\title{
DIRECT MESHLESS KERNEL TECHNIQUES FOR TIME-DEPENDENT EQUATIONS
}

\author{
Y.C. HON AND R. SCHABACK
}

\begin{abstract}
We provide a class of positive definite kernels that allow to solve certain evolution equations of parabolic type for scattered initial data by kernel-based interpolation or approximation, avoiding time intergation completely. Some numerical illustrations are given.
\end{abstract}

Key words. partial differential equation, expansion kernels, heat kernel, Trefftz methods

AMS subject classifications. 65M20, 65M12, 65M22, 35K05, 35K08,35Q79, 45H99

1. Introduction. There are plenty of application papers in which kernels or radial basis functions are successfully used for solving partial differential equations by meshless methods. The usage of kernels is typically based on spatial interpolation at scattered locations, writing the trial functions "entirely in terms of nodes"[2]. For stationary partial differential equations, the discretization can take pointwise analytic derivatives of the trial functions to end up with a linear system of equations. This started in [5] and was pursued in the following years, including a convergence theory in [7]. There are also variations that use weak data, like the Meshless Local PetrovGalerkin method [1] with a convergence theory in [9]. For the potential equation, there are special kernels that allow the use of trial functions that satisfy the differential equation exactly [8,4]. This is a variation of the general idea of Trefftz [12] to use trial functions that satisfy the PDE exactly.

For time-dependent partial differential equations, meshless kernel-based methods were similarly based on a fixed spatial interpolation, but now the coefficients are timedependent, and one obtains a system of ordinary differential equations for these. This is the well-known Method of Lines, sometimes also called differential quadrature, and it turned to be experimentally useful in various cases (see e.g. $[13,6,3,11]$ ). But we follow the Trefftz philosophy here and use special kernels that satisfy a linear evolution-type PDE

$$
u_{t}(x, t)=L u(x, t)
$$

with a purely spatial and elliptic operator $L$ exactly. This will eliminate time integration, but at the expense of using kernels defined via expansions into eigenfunctions of the spatial differential operator $L$. Of course, this is a special case of a spectral method, conveniently stated in terms of a time-dependent positive definite kernel.

We give a rigid error analysis of this technique and provide a few numerical examples.

Instead of using trial functions that satisfy the boundary conditions but violate the differential equation, we approximate the solution by selecting functions that violate the boundary conditions but satisfy the differential equation.

2. Linear Elliptic Equations. We take a spatial domain $\Omega \subset \mathbb{R}^{d}$ and some kind of homogeneous boundary condition on $\partial \Omega$. Then, for a linear self-adjoint elliptic differential operator $L$, we assume to have eigenfunctions $u_{n}$ on $\Omega$ for the associated boundary value problem, i.e.

$$
L u_{n}=\lambda_{n} u_{n} \text { in } \Omega, n \in N
$$


with a countable index set $N$. Our running example will be $L=\Delta$ on $\Omega=[0, \pi]^{d}$ with homogeneous Dirichlet boundary conditions, leading to

$$
u_{k}(x)=\prod_{i=1}^{d} \sin \left(k_{i} x_{i}\right), \lambda_{k}=-\|k\|_{2}^{2}, k \in N:=\mathbb{N}_{0}^{d} \backslash\{0\}
$$

in standard multi-index notation.

A solution of the problem

$$
L u=f
$$

with homogeneous boundary conditions can then be written formally by expanding $f$ into the eigenfunctions as

$$
f=\sum_{n \in N} \alpha_{n} u_{n}
$$

and then writing the solution $u$ as

$$
u=\sum_{n \in N} \frac{\alpha_{n}}{\lambda_{n}} u_{n}
$$

This needs a discussion of convergence of the series. We shall do this in a way that is closely linked to reproducing kernel Hilbert spaces.

3. Expansion Kernels. We now fix positive real numbers $\mu_{n}$ for all $n \in N$ to let an expansion kernel

$$
K_{\mu}(x, y):=\sum_{n \in N} \mu_{n} u_{n}(x) u_{n}(y)
$$

satisfy the summability condition

$$
K_{\mu}(x, x)=\sum_{n \in N} \mu_{n} u_{n}(x)^{2} \leq C^{2}<\infty \text { for all } x \in \Omega .
$$

This kernel is positive semidefinite on $\Omega$, i.e. for all selections of finite point sets $X=$ $\left\{x_{1}, \ldots, x_{M}\right\} \subset \Omega$, the $M \times M$ kernel matrices $A=A(X)$ with entries $K_{\mu}\left(x_{j}, x_{k}\right), 1 \leq$ $j, k \leq M$ are symmetric and positive semidefinite.

By well-known results, such a kernel is reproducing in the Hilbert space $H_{\mu}$ of all functions of the form

$$
f_{c}(x):=\sum_{n \in N} c_{n} u_{n}(x), x \in \Omega
$$

under the condition

$$
\left\|f_{c}\right\|_{\mu}^{2}:=\sum_{n \in N} \frac{c_{n}^{2}}{\mu_{n}}<\infty
$$

related to the inner product

$$
\left(f_{c}, f_{d}\right)_{\mu}:=\sum_{n \in N} \frac{c_{n} d_{n}}{\mu_{n}}
$$


letting the reproduction follow the formula

$$
f_{c}(x)=\left(f_{c}, K_{\mu}(x, \cdot)\right)_{\mu} \text { for all } x \in \Omega, f_{c} \in H_{\mu} .
$$

Note that this gives us a variety of Hilbert spaces, and we shall check now how $L$ maps functions between these spaces. Taking $u \in H_{\mu}$ with coefficients $c_{n}$, we get that $L u$ has coefficients $\lambda_{n} c_{n}$, and thus

$$
L: H_{\mu} \rightarrow H_{\mu / \lambda^{2}}
$$

allows to look at solutions of $L u=f$ for various regularity assumptions. Here, we denote the sequence with values $\frac{\mu_{n}}{\lambda_{n}^{2}}$ by $\mu / \lambda^{2}$ for short.

We require the initial function $u_{0}$ to be in $H$, i.e.

$$
u_{0}(x)=\sum_{n \in N} \gamma_{n} u_{n}(x)
$$

with

$$
\left\|u_{0}\right\|_{H}^{2}=\sum_{n \in N} \frac{\gamma_{n}^{2}}{\mu_{n}}<\infty .
$$

The basic idea now is to construct a time-dependent kernel $K$ satisfying the differential equation exactly. We do this by defining

$$
K(x, y, t):=\sum_{n \in N} \mu_{n}(t) u_{n}(x) u_{n}(y), x, y \in \Omega, t \geq 0
$$

with initial conditions

$$
\mu_{n}(0)=\mu_{n}, n \in N
$$

leading to

$$
K(x, y, 0)=K_{0}(x, y) \text { for all } x, y \in \Omega .
$$

To let the differential equation be satisfied in the sense

$$
K_{t}(x, y, t)=L^{x} K(x, y, t) \text { for all } x, y \in \Omega, t \geq 0
$$

where the superscript $x$ indicates that $L$ acts on the variable $x$, we have to satisfy

$$
\begin{aligned}
\sum_{n \in N} \mu_{n}^{\prime}(t) u_{n}(x) u_{n}(y) & =\sum_{n \in N} \mu_{n}(t) L^{x} u_{n}(x) u_{n}(y) \\
& =\sum_{n \in N} \mu_{n}(t) \lambda_{n} u_{n}(x) u_{n}(y)
\end{aligned}
$$

and this leads to the ordinary differential equations

$$
\mu_{n}^{\prime}(t)=\mu_{n}(t) \lambda_{n}
$$

with the solution

$$
\mu_{n}(t)=\mu_{n} \exp \left(\lambda_{n} t\right), t \geq 0, n \in N \text {. }
$$


Thus our kernel is

$$
K(x, y, t)=\sum_{n \in N} \mu_{n} \exp \left(\lambda_{n} t\right) u_{n}(x) u_{n}(y), x, y \in \Omega, t \geq 0
$$

and in case of positive eigenvalues we need the condition

$$
\sum_{n \in N} \mu_{n} \exp \left(\lambda_{n} T\right) u_{n}(x)^{2}<\infty \text { for all } x \in \Omega
$$

to be able to work in $[0, T]$. This approach generalizes the standard heat kernel. Note that elliptic operators will have negative eigenvalues in (2.1), and then the coefficients $\mu_{n}(t)$ will decay with increasing time.

4. Interpolatory Methods. Since we have a positive semidefinite kernel $K_{0}$ on the spatial domain, we can choose a set $X=\left\{x_{1}, \ldots, x_{M}\right\} \subset \Omega$ of points in $\Omega$ and interpolate the initial function $u_{0}$ by a linear combination of the functions $K_{0}\left(x, x_{m}\right), 1 \leq m \leq M$ via the linear system

$$
u_{0}\left(x_{i}\right)=\sum_{m=1}^{M} \alpha_{m} K_{0}\left(x_{i}, x_{m}\right)
$$

for $1 \leq i \leq M$. If the initial function $u_{0}$ lies in $H$, this problem is solvable, though the kernel matrix is only positive semidefinite. We then define

$$
\tilde{u}(x, t):=\sum_{m=1}^{M} \alpha_{m} K\left(x, x_{m}, t\right)
$$

to see that the differential equation and the boundary conditions are satisfied.

The error satisfies the differential equation and the boundary conditions. Thus the error is exactly the evolution of the initial error under the differential equation. If the maximum principle holds, the error for all positive times is thus bounded by the $L_{\infty}$ interpolation error $\left\|\tilde{u}(\cdot, 0)-u_{0}\right\|_{\infty}$ at startup. A theoretical analysis of this error requires an application of kernel interpolation theory to $K(x, y, 0)$.

The choice of the weights in the kernel series (3.1) will depend on the smoothness of the starting function $u_{0}$, since kernel interpolation theory $[14,10]$ tells us that the smoothness of the kernel $K(x, y, 0)$ should be not lower than the smoothness of the function supplying the data. And since, for example, the smoothness of the functions generated by trigonometric series is related to the decay of the coefficients, the smoothness of $K(x, y, 0)$ will usually be controlled by decay of the $\lambda_{k}$.

Direct interpolation of initial data by linear combinations of eigenfunctions is not possible in general. The use of kernels always allows interpolation.

5. Examples . We start the simple example from (2.2) here.

The choice $\mu_{k}=1 / k$ ! gives a series which generates an analytic kernel plotted in Figure 5.1. It has an explicit representation

$$
\begin{aligned}
& 4 K(x, y, 0)=\quad \exp (\exp (\pi(x+y))) \\
&-\exp (\exp (\pi(x-y)))-\exp (\exp (-\pi(x+y))) \\
&-\exp (-\pi(x-y)))
\end{aligned}
$$

which unfortunately suffers from severe cancellation. But the rapid convergence of the series (3.1) allows to sum the series up until the limit of double precision is reached, 

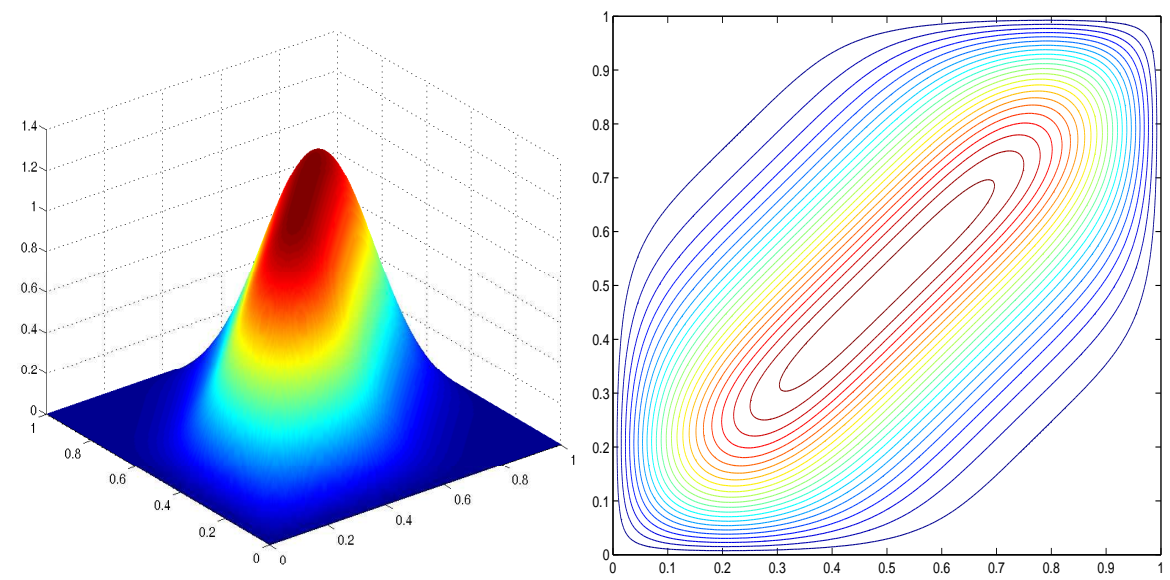

FIG. 5.1. Kernel with weights $1 / n$ !

i.e. at $k=19$. This will, however, lead to inevitable rank loss in (4.1) for more than $n=19$ data points. Nonetheless, and in particular if the initial function $u_{0}$ is very smooth, there usually are good projections of the right-hand side into the column space of the matrix, leading to unexpectedly good results. Figure 5.2 shows an example for the starting function $u_{0}(x)=1-2|x-0.5|$ using only 12 interior points. The error is bounded by the visible difference of the starting function and its first interpolant.

By simple spectral shifts, this example generalizes to the case $L u=\Delta u+\kappa u$, and similarly for other spatial operators that have known eigenfunction expansions.

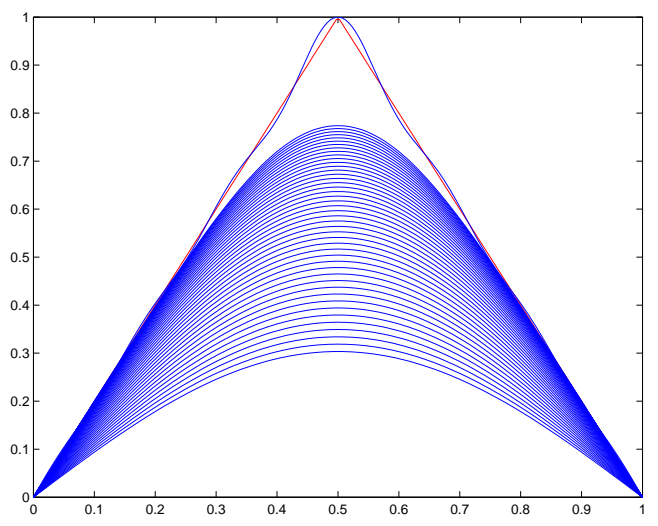

FIG. 5.2. Solution of heat equation

If one tries to solve the heat equation backwards this way, the solution must increase exponentially. Figure 5.3 shows two examples:

- starting with $u_{0}(x)=x(1-x)$ up to time $t=-0.005$ in steps of 0.0001 ,

- starting with $u_{0}(x)=1-2|x-0.5|$ up to time $t=-0.001$ in steps of 0.0001 .

The final example concerns the wave equation. The time-dependent part now is

$$
\mu_{n}(t)=\mu_{n}(0) \cos \left(\lambda_{n} t\right)=\frac{1}{n !} \cos (n \pi t)
$$



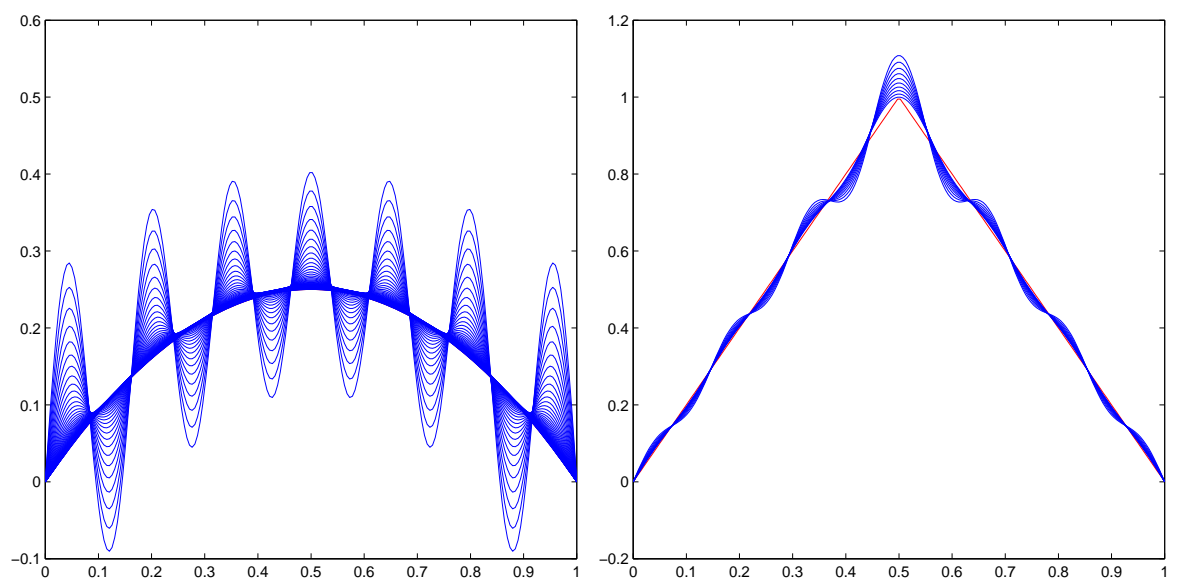

FIG. 5.3. Two backward calculations

in this case, using (2.2) in the spatial variables. The result is in Figure 5.4 for $u_{0}(x)=$ $1-2|x-0.5|$ and times up to $t=1$ in steps of 0.05 . Note that the wave starts with the interpolant and reflects back to it.

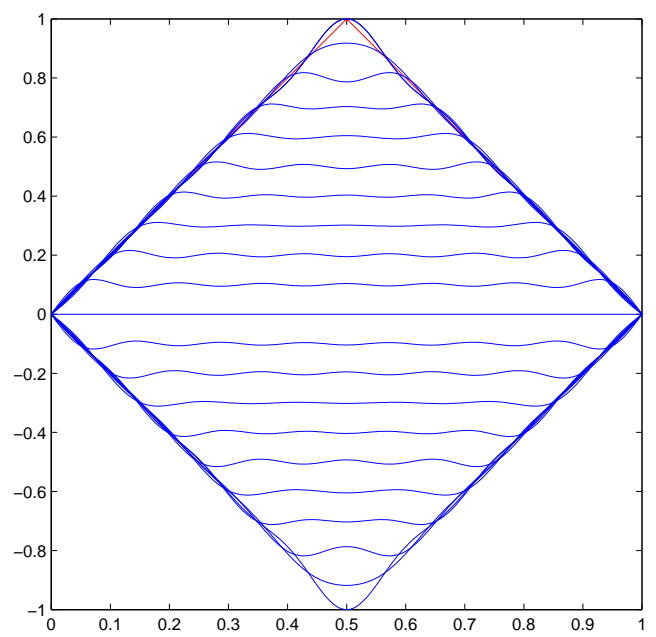

FIG. 5.4. Solution of wave equation

6. Extensions. This approach generalizes to other cases where separation of variables works, e.g. the wave equation. If there is a linear differential operator $D$ acting with respect to time, the problem $D u(x, t)=L u(x, t)$ can be split into eigenvalue problems

$$
D v_{n}(t)=\lambda_{n} v_{n}(t), L u_{n}(x)=\lambda_{n} u_{n}(x),
$$

for appropriate homogeneous boundary conditions, and we can define a kernel

$$
K(x, y, t):=\sum_{n} \mu_{n} u_{n}(x) u_{n}(y) v_{n}(t)
$$


under the summability condition

$$
K(x, x, t)=\sum_{n} \mu_{n} u_{n}^{2}(x)\left|v_{n}(t)\right|<\infty
$$

To make interpolation at $t=0$ work, additional conditions must be satisfied. In case of the wave equation $u_{t t}=\Delta u$, we use trial functions

$$
u(x, t):=\sum_{j=1}^{N} a_{j} K\left(x, x_{j}, t\right)+\sum_{j=1}^{N} b_{j} K_{t}\left(x, x_{j}, t\right)
$$

since for a useful initial-value problem we have to prescribe both $u(x, 0)$ and $u_{t}(x, 0)$. On the spatial domain $[0, \pi]$ we can use $u_{n}(x)=\sin (n x)$ and $v_{n}(t)=\cos (n t)$ to form kernels. We pose interpolation conditions

$$
\begin{aligned}
u\left(x_{k}, 0\right) & =\sum_{j=1}^{N} a_{j} K\left(x_{k}, x_{j}, 0\right)+\sum_{j=1}^{N} b_{j} K_{t}\left(x_{k}, x_{j}, 0\right) \\
& =\sum_{j=1}^{N} a_{j} K\left(x_{k}, x_{j}, 0\right) \\
u_{t}\left(x_{k}, 0\right) & =\sum_{j=1}^{N} a_{j} K_{t}\left(x_{k}, x_{j}, 0\right)+\sum_{j=1}^{N} b_{j} K_{t t}\left(x_{k}, x_{j}, 0\right) \\
& =\sum_{j=1}^{N} b_{j} K_{t t}\left(x_{k}, x_{j}, 0\right)
\end{aligned}
$$

that simplify because of $v_{n}^{\prime}(0)=0$ and thus $K_{t}(x, y, 0)=0$. The kernels $K$ and

$$
K_{t t}(x, y, t)=\sum_{n} \lambda_{n} \mu_{n} u_{n}(x) u_{n}(y) v_{n}(t)
$$

are both definite, and the interpolation problem is solvable.

\section{REFERENCES}

[1] S. N. Atluri and T.-L. Zhu, A new meshless local Petrov-Galerkin (MLPG) approach in Computational Mechanics, Computational Mechanics, 22 (1998), pp. 117-127.

[2] T. Belytschko, Y. Krongauz, D. Organ, M. Fleming, and P. Krysl, Meshless methods: an overview and recent developments, Computer Methods in Applied Mechanics and Engineering, special issue, 139 (1996), pp. 3-47.

[3] M. Dehghan And A. Shokri, A meshless method for numerical solution of the onedimensional wave equation with an integral condition using radial basis functions, Numerical Algorithms, 52 (2009), pp. 461-477. 10.1007/s11075-009-9293-0.

[4] Y. Hon And R. Schaback, Solving the 3D Laplace equation by meshless collocation via harmonic kernels. to appear in Adv. in Comp. Math., 2010.

[5] E. J. Kansa, Application of Hardy's multiquadric interpolation to hydrodynamics, in Proc. 1986 Simul. Conf., Vol. 4, 1986, pp. 111-117.

[6] E. J. Kansa, H. Power, F. G. E., And L. Ling, A volumetric integral radial basis function method for time-dependent partial differential equations $i$, Engineering Analysis with Boundary Elements, 28 (2004), pp. 1191-1206.

[7] R. Schaback, Convergence of unsymmetric kernel-based meshless collocation methods, SIAM J. Numer. Anal., 45 (2007), pp. 333-351 (electronic).

[8] — Solving the Laplace equation by meshless collocation using harmonic kernels, Adv. in Comp. Math., 31 (2009), pp. 457-470. DOI 10.1007/s10444-008-9078-3. 
[9] — Unsymmetric meshless methods for operator equations, Numer. Math., 114 (2010), pp. 629-651.

[10] R. Schaback and H. Wendland, Kernel techniques: from machine learning to meshless methods, Acta Numerica, 15 (2006), pp. 543-639.

[11] Q. Shen, A meshless method of lines for the numerical solution of kdv equation using radial basis functions, Engineering Analysis with Boundary Elements, 33 (2009), pp. 1171-1180.

[12] E. Trefftz, Ein Gegenstück zum Ritzschen Verfahren, in 2. Int. Kongr. f. Techn. Mechanik, Zürich, 1926, pp. 131-137.

[13] M. WANG, X. WANG, AND D. Guo, A level set method for structural topology optimization, Comput Methods Appl. Mech. Eng., 192 (2003), pp. 227-246.

[14] H. Wendland, Scattered Data Approximation, Cambridge University Press, 2005. 